\title{
Prognostic Factors After Hepatectomy for Gastric Adenocarcinoma Liver Metastases: Desmoplastic Growth Pattern as the Key to Improved Overall Survival
}

This article was published in the following Dove Press journal:

Cancer Management and Research

\author{
Maria José Temido ${ }^{1,2, *}$ \\ Rui Caetano Oliveira ${ }^{3-6, *}$ \\ Ricardo Martins ${ }^{2,4,5,7}$ \\ Marco Serôdio iD ${ }^{2,7}$ \\ Beatriz Costa iD $^{2,5,7}$ \\ César Carvalho \\ Eva Santos ${ }^{2,7}$ \\ Luís Ferreira $^{7}$ \\ Paulo Teixeira (iD) 3,8 \\ Maria Augusta Cipriano ${ }^{3}$ \\ José Guilherme Tralhão ${ }^{2,4,5,7}$ \\ Henrique Alexandrino ${ }^{2,5,7}$
}

'Gastroenterology Department, Centro Hospitalar e Universitário de Coimbra, Coimbra 3000-075, Portugal; ${ }^{2}$ Faculty of Medicine, University of Coimbra, Coimbra 3000-548, Portugal; ${ }^{3}$ Pathology Department, Centro Hospitalar e Universitário de Coimbra, Coimbra 3000-075, Portugal; ${ }^{4}$ Biophysics Institute, Faculty of Medicine, University of Coimbra, Coimbra 3000-548, Portugal;

${ }^{5}$ Coimbra Institute for Clinical and Biomedical Research (iCBR), Area of Environment Genetics and Oncobiology (CIMAGO), Faculty of Medicine, University of Coimbra, Coimbra 3000-548, Portugal; ${ }^{6}$ Faculdade de Medicina da Universidade do Porto, Porto 4200-319, Portugal; ${ }^{7}$ Department of Surgery, Centro Hospitalar e Universitário de Coimbra, Coimbra 3000-075, Portugal; ${ }^{8}$ Department Biomedical Laboratory Sciences, ESTeSCCoimbra Health School, Polytechnic Institute of Coimbra, Coimbra 3046-854, Portugal

*These authors contributed equally to this work

Correspondence: Maria José Temido Centro Hospitalar e Universitário de Coimbra, Praceta Professor Mota Pinto, Coimbra 3004-56I, Portugal Tel +351910772166

Email mariajosetemido@gmail.com
Purpose: Hepatectomy $(\mathrm{Hp})$ is an alternative approach for the treatment of gastric carcinoma liver metastases (GCLM). However, prognostic factors that may assist patient selection are still controversial. Several pathologic features, such as the growth pattern (GP), associated with prognosis in colorectal cancer liver metastases, were never investigated in GCLM. Our principal aim was to assess if the GP has prognostic impact on GCLM.

Patients and Methods: Review of the clinical and pathological characteristics of 19 consecutive patients submitted to surgical resection of GCLM with curative intent at our department. Major potential prognostic factors considered were patients' gender, age, timing and extent of Hp, postoperative course, as well as histopathological characteristics of primary and secondary tumors.

Results: Major morbidity occurred in four patients, mortality in one. Median and 5-year overall survival were 17 months and $26.7 \%$, respectively. Ten patients developed recurrent disease and two patients survived more than 10 years. Factors independently associated with overall survival were the absence of major morbidity, distal location of the primary tumor, and desmoplastic GP $(\mathrm{p}<0.05)$.

Conclusion: The selection of patients is crucial for the improvement of survival rates of GCLM. Consequently, we demonstrate for the first time that the desmoplastic GP of GCLM is associated with improved outcomes, prompting further research on tumor-host interactions.

Keywords: liver metastases, gastric carcinoma, hepatectomy, growth pattern, prognosis

\section{Introduction}

Gastric carcinoma (GC) is the third leading cause of cancer-related death. ${ }^{1}$ The aggressiveness of this malignancy is associated with high metastatic rates. ${ }^{2}$

Liver metastases (LM) are found in up to $37 \%$ of the patients with GC after curative gastrectomy. ${ }^{3}$ Despite the fact that chemotherapy is the recommended treatment in advanced and recurrent GC, it does not achieve long survival. ${ }^{4-6}$ Recent studies support hepatectomy (Hp) as an alternative in selected patients with GCLM, with promising results of 5-year survival rates of about $30 \% .^{7-10}$ However, $\mathrm{Hp}$ in the treatment of gastric carcinoma liver metastases (GCLM) is still controversial. $^{6}$

Many studies aimed at validating clinical factors associated with longer survival, as a result of more indolent biology of the tumor. ${ }^{8,11-18}$ Other important 
pathologic factors, either tumor-related, host-related, or dependent upon the tumor-host interaction, may also be relevant. Examples of these features include tumor thickness at the tumor-normal interface (TTNI) and tumor regression grade (TRG) that have emerged in the setting of colorectal cancer liver metastases (CRLM). ${ }^{19,20}$ Additionally, HER-2 expression has been associated with more aggressive behavior in GC although its prognostic value is still controversial. ${ }^{21}$ The growth pattern (GP) of metastases, reflecting distinct characteristics of the tumor microenvironment, also constitutes a potential prognostic factor. GP has shown to be relevant in $\mathrm{CRLM}^{22-25}$ and seems to represent the path to pursue in the selection of patients for Hp. ${ }^{10,26}$

In our study, we sought to investigate both clinical and histopathological factors, with emphasis on GCLM's GP, potentially associated with an improved outcome after Hp. In the future, these factors may increase the patients' selection accuracy for an aggressive surgical approach to liver-only metastatic GC.

\section{Patients and Methods}

\section{Study Design}

Review of clinical and pathological factors of the patients that underwent surgical resection of GCLM with curative intent between February-1997 and November-2017. In our center indications for $\mathrm{Hp}$ in GCLM were decided after multidisciplinary meeting and were: acceptable performance status; good hepatocellular function; previous or predicted curative resection of the gastric primary; no documented extrahepatic metastatic disease and possibility of achieving hepatic margin-free resection with minimal morbimortality. Furthermore, patients with oligometastatic disease were preferred as these were thought to have less aggressive disease. More recently, we have selected cases of good biological (clinical evolution and serum markers) and radiological response to neoadjuvant chemotherapy, meaning that we have given preference to metachronous resection, even when the presentation was synchronous. Clinical information was collected from patients' medical records and hospital's database. The study was approved by the institutional ethics committee (number CHUC-12719). Written informed consent was signed by the patient or its legal representative. The project was subjected to the standards of good clinical practice and always complied with the ethical precepts of the Helsinki's Declaration.
Confidentiality of the data was respected and maintained at all times.

\section{Study Population}

The population included 19 patients, 13 men and six women (Table 1). The mean age was $66.3 \pm 9.9$ years (range 44-79 years); 14 (73.7\%) patients were older than 60 years.

The study only included patients submitted to hepatic resection with curative intent. Patients with multiple (more than five) liver metastases, extra-hepatic metastatic disease and low-performance status were excluded.

Metastases were diagnosed synchronously in 16 $(84.2 \%)$ patients. In seven of these patients' $(43.8 \%)$ gastrectomy and $\mathrm{Hp}$ were simultaneous. The mean interval between diagnosis and $\mathrm{Hp}$ was $5.1 \pm 6.2$ months and the mean interval between gastrectomy and $\mathrm{Hp}$ was 6.2 \pm 6.7 months. The median number of liver metastases was 1 (range 1-4). A bilobar distribution was found in three patients $(15.8 \%)$. The mean size of metastases was 33.7 $\pm 23.8 \mathrm{~mm}$.

The primary tumor was multiple in only one $(5.3 \%)$ patient - in cardia and antrum - and single in 18 cases $(94.7 \%)$. In the latter group, the GC was located in the gastro-esophageal junction (GEJ) and fundus in three $(16.6 \%)$ patients, in the body in ten $(55.6 \%)$ and, in the antrum in five $(27.8 \%)$. The tumors in GEJ were all Siewert type III. The mean largest diameter was 5.8 $\pm 2.5 \mathrm{~cm}$ (range 1.5-10.5). Lymph node metastases were absent (N0) in four (21.1\%) patients and present in 15 $(78.9 \%)$ as follows: $\mathrm{N} 1$ in two $(10.5 \%)$, N2 in six $(31.6 \%)$ and $\mathrm{N} 3$ in seven $(36.8 \%)$.

As far as the primary tumor is concerned, neoadjuvant chemotherapy was given to two (10.5\%) patients. Adjuvant therapies were performed either as chemotherapy in $12(63.2 \%)$ patients and as radiotherapy in one (5.3\%). For the LM, neoadjuvant chemotherapy was used in $13(68.4 \%)$ patients. A total of eight patients $(42.1 \%)$ were given adjuvant chemotherapy after Hp.

The Association Française de Chirurgie (AFC) Score was calculated according to Adam et al with a median value of 5 (range $4-9){ }^{27}$

\section{Operative Procedures}

Our department's technique for $\mathrm{Hp}$ has been previously described. $^{28}$ Major Hp was performed in three $(15.8 \%)$ patients. The resection was anatomical in ten $(52.6 \%)$ cases (Table 1). No laparoscopic Hp was performed. 
Table I Clinical and Pathological Characteristics of the Study Population ( $\mathrm{N}=19$ Patients Undergoing Curative Hepatectomy for Gastric Carcinoma Liver Metastases)

\begin{tabular}{|l|l|}
\hline Parameters & No. of Patients (\%) \\
\hline Gender & $13(68.4 \%)$ \\
\hline $\begin{array}{l}\text { Male } \\
\text { Female }\end{array}$ & $6(31.6 \%)$ \\
\hline Age & $14(73.7 \%)$ \\
\hline$\leq 60$ & $5(26.3 \%)$ \\
$>60$ &
\end{tabular}

Neoadjuvant chemotherapy before hepatectomy

\begin{tabular}{|l|l|}
\hline Yes & $\begin{array}{l}13(68.4 \%) \\
6(31.6 \%)\end{array}$ \\
\hline
\end{tabular}

Timing of diagnosis

\begin{tabular}{|c|c|}
\hline $\begin{array}{l}\text { Synchronous } \\
\text { Metachronous }\end{array}$ & $\begin{array}{l}16(84.2 \%) \\
3(15.8 \%)\end{array}$ \\
\hline \multicolumn{2}{|c|}{ Interval between surgeries } \\
\hline$\leq 8$ months & $10(52.6 \%)$ \\
\hline$>8$ months & 9 (47.4\%) \\
\hline \multicolumn{2}{|c|}{ Extension of hepatectomy } \\
\hline Minor & $16(84.2 \%)$ \\
\hline Major & 3 (I5.8\%) \\
\hline
\end{tabular}

\section{Postoperative course}

\begin{tabular}{l|l} 
Major morbidity & $5(26.3 \%)$ \\
No or minor morbidity & $14(73.7 \%)$
\end{tabular}

\begin{tabular}{|l|l|}
\hline Location of primary tumor \\
\hline $\begin{array}{l}\text { Proximal } \\
\text { Distal }\end{array}$ & $\begin{array}{l}4(21.1 \%) \\
15(78.9 \%)\end{array}$ \\
\hline Lauren type & $3(15.8 \%)$ \\
\hline Diffuse and undetermined & $16(84.2 \%)$ \\
\hline Intestinal &
\end{tabular}

\section{Histologic type}

\begin{tabular}{|l|l|}
\hline $\begin{array}{l}\text { Papillary and NOS } \\
\text { Other }\end{array}$ & $\begin{array}{l}\text { II (57.9\%) } \\
8(42.1 \%)\end{array}$ \\
\hline Grading of gastric lesion & $16(84.2 \%)$ \\
\hline GI and G2 & $3(15.8 \%)$ \\
G3 & \multicolumn{2}{|l}{} \\
\hline Depth of invasion & $3(15.8 \%)$ \\
\hline ST2 & $16(84.2 \%)$ \\
$>$ T2 &
\end{tabular}

(Continued)
Table I (Continued).

\begin{tabular}{|c|c|}
\hline Parameters & No. of Patients (\%) \\
\hline \multicolumn{2}{|c|}{ Lymph node metastases } \\
\hline No & $4(21.2 \%)$ \\
\hline $\mathrm{N}+$ & 15 (78.9\%) \\
\hline \multicolumn{2}{|l|}{ Lymph node ratio } \\
\hline$<0.5$ & $13(76.5 \%)$ \\
\hline$\geq 0.5$ & $4(23.5 \%)$ \\
\hline \multicolumn{2}{|l|}{ Growth pattern } \\
\hline Desmoplastic & $6(35.3 \%)$ \\
\hline Non-desmoplastic & II (64.7\%) \\
\hline
\end{tabular}

\section{Morbidity and Mortality}

Postoperative morbidity was defined up to the first 90 days after surgery, graded according to Dindo-Clavien classification. $^{29}$ Posthepatectomy liver failure (PHLF) was defined in concordance with the "50-50 Criteria",30 and its severity was graded as proposed by Rahbari et al. ${ }^{31}$ Biloma and bile leakage and posthepatectomy hemorrhage were defined according to consensus definitions. ${ }^{32,33}$

\section{Histopathological Analysis}

Archival tumor material was reviewed by two experienced pathologists (MAC and RCO) blinded to clinical data and outcomes.

\section{Primary Tumor}

The behavior of the GC was evaluated according to size, location, resection margins, Lauren classification, World Health Organization (WHO) classification, ${ }^{34}$ depth of invasion, infiltrative pattern, grading, inflammatory response and lymphatic and vascular invasion. All GCs were staged or restaged according to TNM classification, 8th edition. ${ }^{34}$

The characteristics of the GC (size, location, macroscopic types, infiltrative patterns and capillary invasion) were categorized according to Japanese classification, 3rd edition from the Japanese Gastric Cancer Association. ${ }^{35}$ Moreover, the histologic types were divided into tubular, papillary, mucinous, mixed, poorly cohesive and, non-otherwise specified (NOS). ${ }^{34}$

Human Epidermoid Receptor (HER)-2's expression in the primary tumor was detected by immunohistochemistry and graded into 0 and $1+$ (negative), 2+ (equivocal) and $3+$ (positive - HER-2 overexpression). ${ }^{34}$ 


\section{Metastases}

The analysis of the metastases' specimens was based on the size of the largest lesion, resection margins, GP, inflammatory response, TRG, TTNI and, HER-2 status. Resection margins were characterized, similarly as in primary tumor into: R0, R1 and, R2. The study of the GP was based on the classification of Vermeulen et $\mathrm{al}^{22}$ for CRLM and categorized into four types: desmoplastic (if a line of stroma with lymphocytes between tumor cells and nontumoral liver parenchyma was present); pushing (if tumor compresses surrounding hepatocytes leading to their narrowing forming a plate of elongated cells with a mild inflammatory infiltrate); replacement (if neoplastic cells only cover the space left by destroyed hepatocytes as a result of the presence of the tumor); or mixed (if more than one pattern was present). TRG and TTNI were assessed as previously described. ${ }^{23}$ HER-2 analysis in the metastases was similar to the primary tumors.

\section{Statistical Analysis}

The data were analyzed with SPSS $^{\text {MM }}$ (version 24.0 for Windows). Survival studies were performed with KaplanMeier curves and compared with the Log rank test. Cox regression was used for multivariate analysis in which the factors used were those with a $\mathrm{p}<0.1$ in the univariate analysis. A p-value of $<0.05$ was considered statistically significant.

Overall survival (OS) was defined as the time interval between Hp and the patient's death or the last record of the patient being alive. Recurrence was defined as the

A

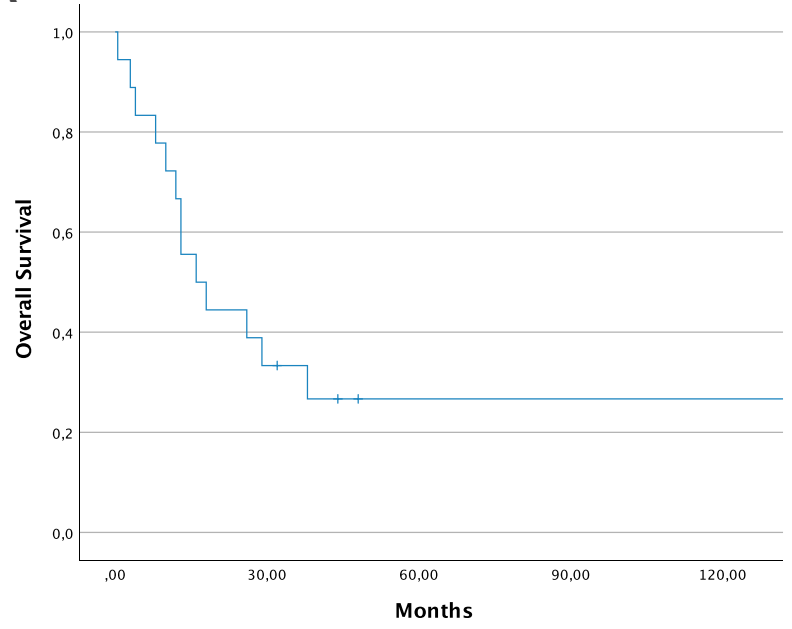

reappearance of malignant lesions either detected by imaging and/or raised tumor markers. Disease-free survival (DFS) was measured beginning in the $\mathrm{Hp}$ and ending at recurrence or death.

\section{Results \\ Early Postoperative Outcomes}

Postoperative mortality occurred in one patient (5.3\%) after total gastrectomy with distal esophagectomy and hepatic segmentectomy due to anastomotic leakage, causing severe sepsis and multiple organ dysfunction. Major morbidity (Dindo grades IIIa-IVb) was observed in four (21.1\%) patients (namely biliary fistula, biloma, pleural effusion and, hemoperitoneum in one case each). Minor morbidity (Dindo grades I and II) was observed in two (10.5\%): superficial surgical site infection and intraperitoneal abscess. The median length of stay was 10 days (range 4-35).

\section{Overall and Disease-Free Survivals}

The median follow-up period was 17 months (range 0.5-135 ) and 3 - and 5-year OS rates were $33.3 \%$ and $26.7 \%$, respectively (Figure 1). Median OS was 17 (range 0.5-135) months. Two patients survived more than ten years after Hp.

Three- and 5-year DFS were 23.8\% (Figure 1). Recurrent disease was diagnosed in ten $(52.6 \%)$ patients - hepatic in eight $(42.1 \%)$, cutaneous in one $(5.3 \%)$ and peritoneal in one $(5.3 \%)$. Recurrent lesions were detected in the first semester after the Hp in six (31.6\%) cases. There was one case $(5.3 \%)$ of rehepatectomy to resect an intra-hepatic recurrence.

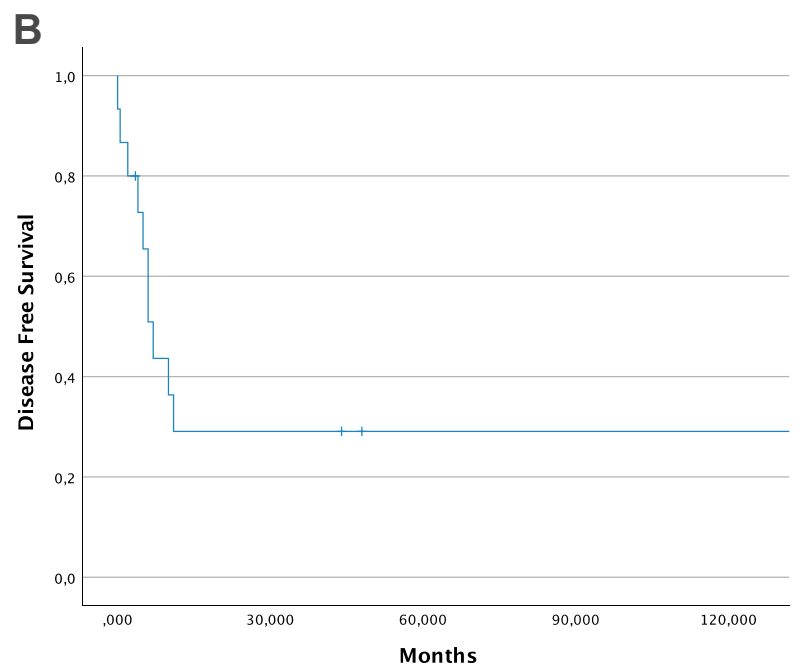

Figure I Kaplan-Meier curves of overall survival (OS) and disease-free survival (DFS) in 19 patients undergoing hepatectomy for gastric cancer liver metastases (GCLM). (A) OS of $26.7 \%$ at 5 years and (B) DFS of $23.8 \%$ at 5 years in the study population. 
Tumoral recurrence led to the death in eight $(42.1 \%)$ patients; only one (5.3\%) died of other causes; the cause of death was unspecified (or not clarified) in six (31.6\%) patients. In the last follow-up four $(21.1 \%)$ patients were alive and only one (5.3\%) had developed recurrent disease.

\section{Histopathological Findings}

The extent of the gastrectomy was determined as R0 in 18 $(94.7 \%)$ cases and as R1 in only one (5.3\%). In the metastatic lesions, margins were negative in 11 patients (57.9\%), R1 in five (26.3\%) and, R2 in one (5.3\%).

HER-2 was considered positive in one primary tumor $(10 \%)$ and in four $(30.1 \%)$ hepatic lesions. In two of these patients, this receptor was overexpressed in the metastases while being negative in GC.
Regarding the GP, the replacement pattern was observed in six (31.6\%), pushing in five $(26.3 \%)$ and desmoplastic in six (31.5\%) (Figure 2). There was one (5.3\%) case of complete tumoral regression after neoadjuvant chemotherapy (with partial radiological response) that was excluded from our survival analysis as the scoring is not possible according to Van Dam et al. ${ }^{25}$

\section{Impact of Clinical Factors in the Disease-Free and Overall Survival}

Univariate analysis showed that the absence of major morbidity (HR 13.183, p<0.001) and more than eight months between gastrectomy and Hp (HR 5.516, $\mathrm{p}=0.019$ ) were strong predictors of longer OS (Figure 3, Supplementary Table).
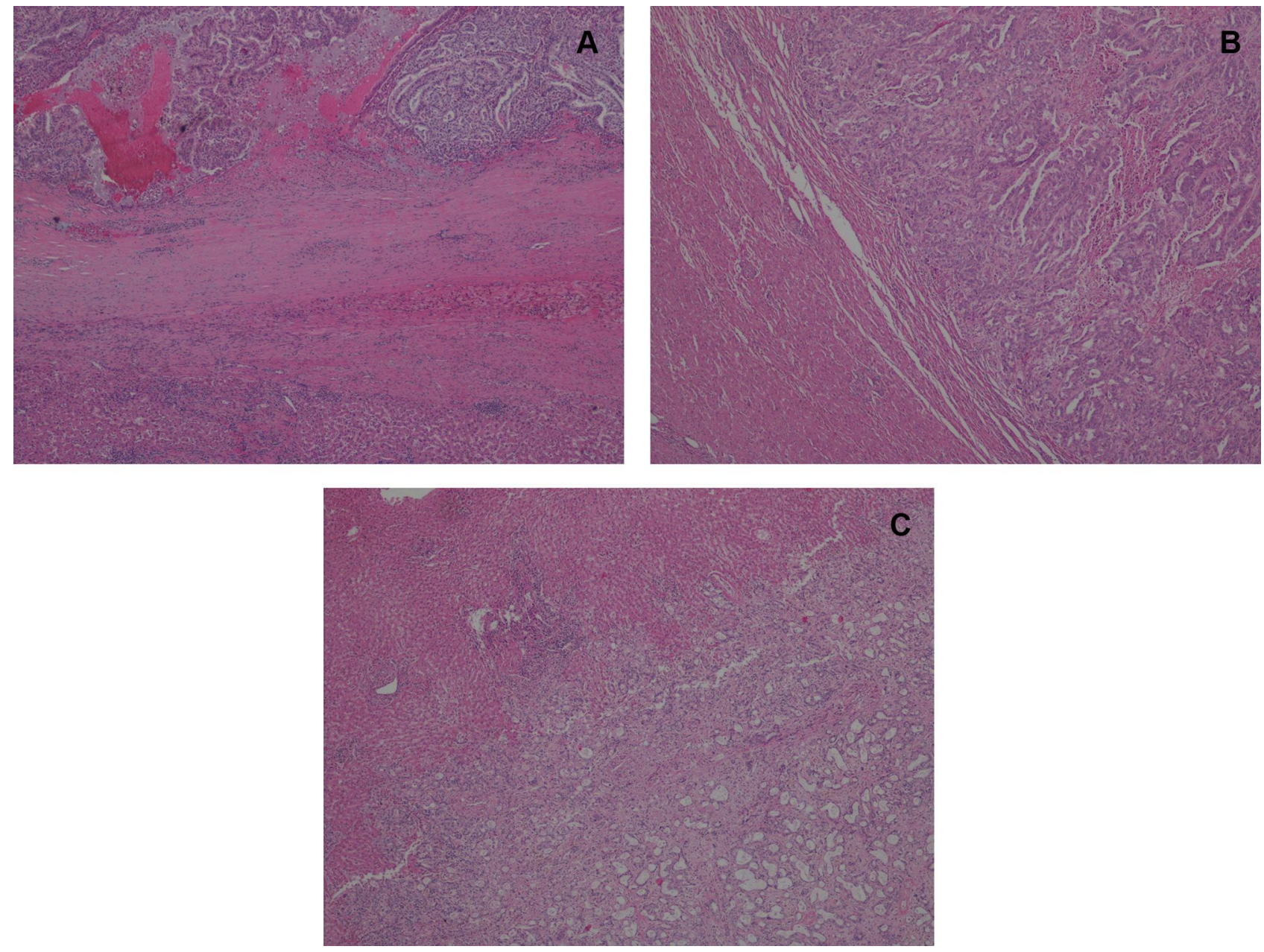

Figure 2 Growth patterns of gastric carcinoma liver metastases. (A) Desmoplastic pattern (a line of fibrotic tissue separates the tumor and the normal parenchyma), H\&E40x. (B) Pushing pattern (tumor compresses the surrounding hepatocytes disrupting the parenchyma), H\&E40x. (C) Replacement pattern (tumor infiltrates the liver without disruption of the parenchyma) H\&E40x. 
DFS was positively affected by metachronous resection (HR 4.064, $\mathrm{p}=0.044$ ) and interval between surgeries longer than eight months (HR 8.505, $\mathrm{p}=0.004)$.

\section{Impact of Histopathological Factors in the Overall and Disease-Free Survival}

Univariate analysis revealed that factors associated with a better OS, regarding the primary tumor, were: location in the body and antrum (HR 8.065, $\mathrm{p}=0.005$ ); papillary and NOS histologic types (HR 5.845, $\mathrm{p}=0.016$ ); Lauren intestinal type (HR 13.333, $\mathrm{p}<0.001)$; T1 and T2 (HR 5.997, $\mathrm{p}=0.014$ ) and low grade (HR 5.113, $\mathrm{p}=0.024$ ) (Figure 3). As far as the metastases are concerned, the positive predictors of

A

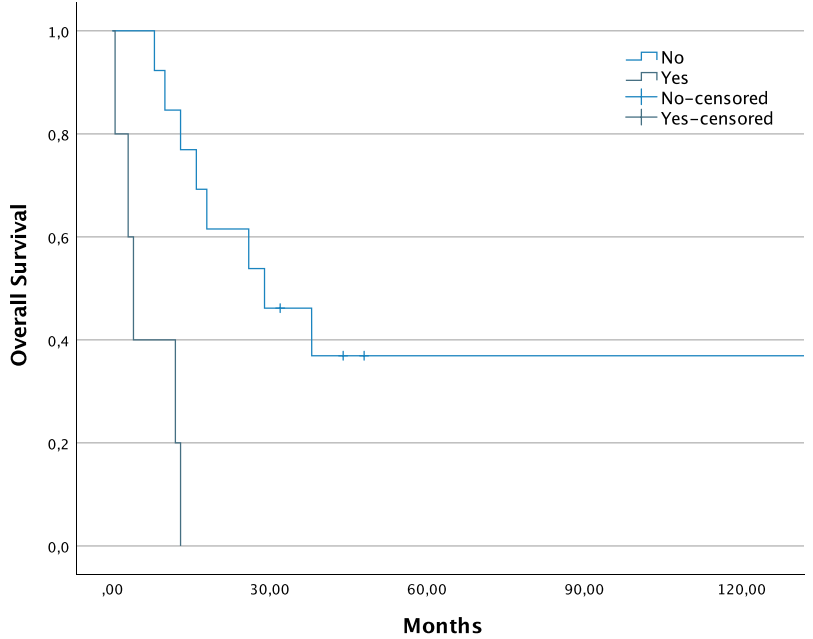

C

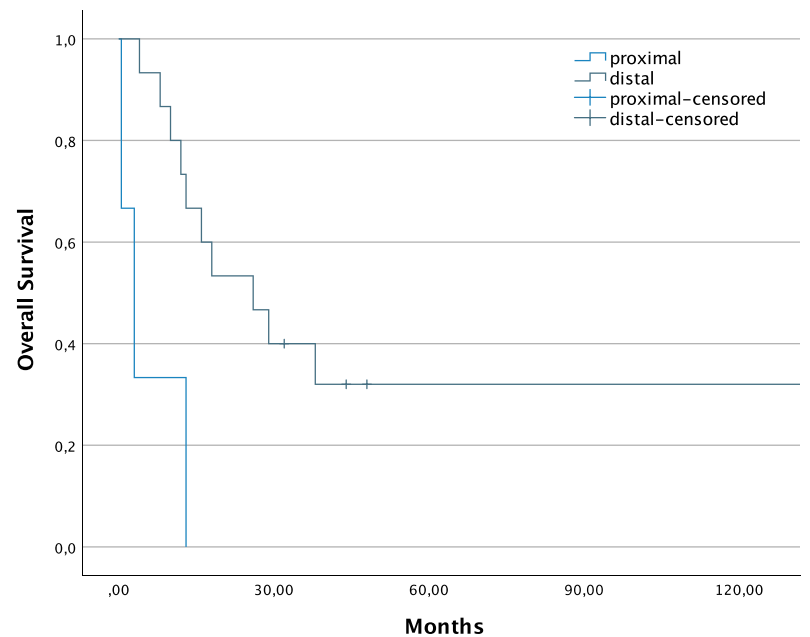

longer survival were: largest lesion smaller or equal to $20 \mathrm{~mm}$ (HR 3.927, $\mathrm{p}=0.048$ ) and desmoplastic GP (HR 4.741, $\mathrm{p}=0.029$ ) (Figure 3).

Papillary and NOS histologic subtypes (HR 11.895, $\mathrm{p}=0.001$ ), $\mathrm{T} 1$ and T2 (HR 4.749, $\mathrm{p}=0.029$ ), lymph node ratio $<0.5$ (HR 4.076, $\mathrm{p}=0.043$ ), desmoplastic GP of metastases (HR 5.013, $\mathrm{p}=0.025$ ) and metastases smaller or equal to $20 \mathrm{~mm}$ (HR $3.871 \mathrm{p}=0.049$ ) were significant prognostic factors for better DFS.

\section{Independent Predictive Negative Factors of Overall Survival}

On multivariate analysis, the absence of major morbidity, location of the primary tumor in body and antrum and,

\section{B}

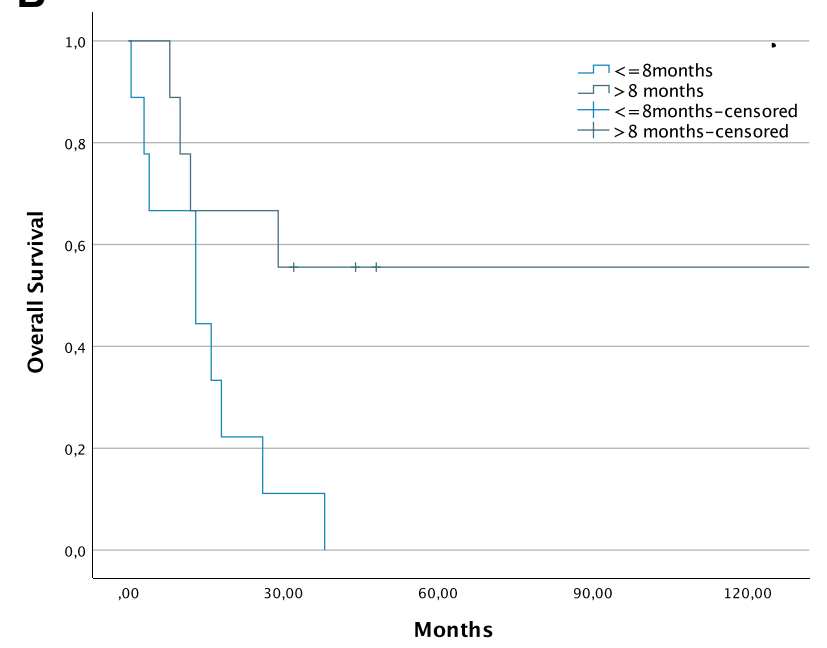

D

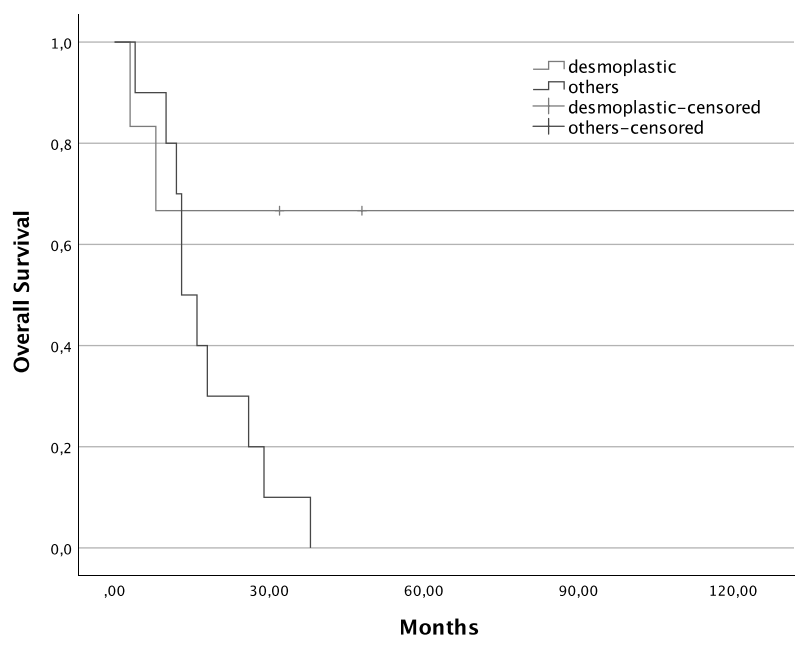

Figure 3 Kaplan-Meier curves of overall survival (OS) in 19 patients undergoing hepatectomy (Hp) for gastric cancer liver metastases (GCLM). (A) OS according to the absence of major morbidity ( 5 -year OS $=36.9 \%$ versus no reported survivors at 5 years, Log rank $\mathrm{p}<0.00 \mathrm{I}$ ). (B) OS according to the interval gastrectomy/Hp higher than eight months (5-year OS $=55.6 \%$ versus no reported survivors at 5 years, Log rank $p=0.019)$. (C) OS according to the location of primary tumor in the body and antrum versus in the fundus or gastro-esophageal junction ( 5 -year OS $=32 \%$ versus no reported survivors at 5 years, Log rank $p=0.005)$. (D) OS according to desmoplastic type of growth pattern of the liver metastases $(5$-year OS $=66.7 \%$ versus no reported survivors at 5 years, Log rank $p=0.04$ ). 
Table 2 Independent Predictors of Overall Survival (Cox Regression) in 19 Patients Undergoing Hepatectomy for Gastric Carcinoma Liver Metastasis

\begin{tabular}{|l|l|l|l|}
\hline & HR & $\mathbf{9 5 \%} \mathbf{~ C l}$ & $\mathbf{P}$ \\
\hline Absence of major morbidity & 0.102 & $0.01-0.84$ & 0.034 \\
Distal primary tumor & 0.075 & $0.01-0.52$ & 0.009 \\
Intestinal primary tumor & 0.01 & $0.0002-0.73$ & 0.035 \\
Desmoplastic growth patterns & 0.1 & $0.01-0.69$ & 0.02 \\
\hline
\end{tabular}

Abbreviations: $\mathrm{HR}$, hazard ratio; $95 \% \mathrm{Cl}, 95 \%$ confidence interval.

desmoplastic GP of LM were independent predictive factors of longer OS (Table 2).

\section{Discussion}

Hepatic resection is valid for selected patients with GCLM, but factors associated with improved survival are still under scrutiny. ${ }^{10}$ This is confirmed by the median OS and 5-year survival rate of our retrospective cohort, 17 months and $26.7 \%$, respectively, similar to previous studies. ${ }^{12,16,18}$ These results are outstanding when compared to the seven months median OS of a study that analyzed the outcomes of GCLM that did not undergo resection. ${ }^{6}$

Regarding the clinical prognostic factors, we reported that an interval between resections of over eight months was associated with improved OS, possibly reflecting patient selection through the response to neoadjuvant chemotherapy. A recent meta-analysis of Montagnani et al ${ }^{18}$ found that metachronous appearance was associated with longer OS consistent with previous studies. ${ }^{36,37}$ On contrary, a systematic review of Markar et al, ${ }^{16}$ as well as several other studies, ${ }^{7,11,13,17}$ failed to prove that metachronous resection was a determinant of prognosis. All these studies showed that solitary metastases and minor resections were predictors of better survival rates. Unexpectedly, these features had no statistical significance in our study. However, this is in accordance with our results on improved survival if the largest metastases were smaller than $20 \mathrm{~mm}$, which was confirmed by Kinoshita et $\mathrm{al}^{7}$ and Ohkura et al. ${ }^{15}$ This likely supports that patients with a low-burden metastatic disease, with an indolent course, are the best candidates for Hp.

Posthepatectomy morbidity has already been validated as a poor prognostic factor after resection for CRLM. ${ }^{38}$ Nevertheless, this has not been investigated in most studies regarding GCLM, with the notable exception of Tatsubayashi et al. ${ }^{8}$ In our cohort, major morbidity proved to be a main determinant of worse long-term outcomes which could be explained by the fact that it delays or even impedes adjuvant chemotherapy. Furthermore, the hypoxic and inflammatory environment could favor the development of micrometastatic disease..$^{39,40}$

An innovative feature of our study is the concurrent investigation of both primary tumor and metastases' main pathologic features, including some that had never been investigated before in the setting of GCLM. ${ }^{10}$

Concerning histopathological analysis of the GC the major predictive factors of a better course were Lauren's intestinal type, better differentiation, proximal location and depth of primary tumor, according with previous observations. ${ }^{7,11,12,17,18}$ Surprisingly, the margin status of metastases and TRG were not associated with prognosis.

One of the most interesting findings was related to HER-2. Detection of the HER-2 expression may have a role in the treatment of GCLM patients as it allows targeted therapy. The thought-provoking result of different expressions between primary tumor and metastases may result from the fact that HER-2, in GC, has a heterogeneous expression - being overexpressed in some areas and absent in others. Another cause to this discrepancy may be different HER-2 expression between locations with overexpression in metastases of negative primary tumors (spatial and temporal heterogeneity).

The growth pattern has already been widely studied in CRLM and recently has shown a correlation with prognosis in LM of uveal melanoma. ${ }^{41}$ We believe that the originality of our study relies on being the first report of this promising prognostic factor in GCLM. Desmoplastic GP was independently associated with a better OS resembling the reality of CRLMs. ${ }^{42}$ This reinforces the need for expanded knowledge on tumor-host interaction as this growth pattern is likely linked to a more intense immunologic reactivity of the host against the tumor. The host does not act as a passive entity, but, instead, tries to contain the metastatic spread. According to Vermeulen et al, ${ }^{22}$ this GP is associated with a pro-apoptotic state as it is characterized by two major defense mechanisms: less proportion of endothelial cells - when compared to pushing and replacement patterns - leading to activation of programmed cell death in tumor cells; and high density of tumor-infiltrating lymphocytes that, together with the hepatocytes near the rim of fibrotic tissue, express high levels of Fas-ligand, an apoptosis marker. Apoptosis, not only of the tumor cells but also of the host cells surrounding the metastases, contributes to a more indolent phenotype and protects against the dissemination of the 
malignancy. Moreover, there is a stroma reaction that builds up a pseudocapsule (Figure 2). These defense mechanisms may be decisive in prognosis, not only because they reflect enhanced immunity against the tumor but also, they may allow a smaller resection margin contributing to a potentially curative Hp. However, this remains to be proven.

Future challenges include investigation of sensitive imaging markers for preoperative identification of GP, particularly with Magnetic Resonance Imaging, especially with desmoplastic GP where a dense rim surrounding the tumor may be identified. ${ }^{23,26}$

Another possible approach may be a detailed analysis of the GC characteristics and molecular classification, ${ }^{26}$ which would allow an upfront stratification of the patients and individualized follow-up. However, tumors undergoing spatial and temporal alterations and selective pressure induced by adjuvant therapies may completely switch the phenotypical findings. ${ }^{43}$ Further studies are needed to address this issue.

A more promising perspective would be the determination of the GP on a liver biopsy, since it would allow direct assessment of the tumoral tissue. Since non-desmoplastic GP is more aggressive, it is expectable a higher expression of stem cell markers, ${ }^{44}$ P53 activation, ${ }^{45}$ Her-2 overexpression and even differences in angiogenesis assessed by CD31 expression ${ }^{46}$ - desmoplastic GP is more angiogenic whereas non-angiogenic metastases grow by mechanisms of vessel co-option. ${ }^{47}$ These findings are highlighted in the study from Lazaris et al where they describe a continuum between tumoral and sinusoidal vessels in the replacement pattern, while in the desmoplastic pattern, the tumor vessels are continuous with arterioles. ${ }^{46}$ Regarding immune composition, a study from 2015 reports a higher UPAR and CD68 expression in the desmoplastic pattern of patients with colorectal cancer liver metastases submitted to neoadjuvant therapy and lower CD3 expression in patients with mixed patterns. $^{48}$

These findings are perfectly translated to a biopsy. Immunohistochemistry evaluation prompts an easy and reproducible method available in the majority of institutions and, should be able to provide some insights into the $\mathrm{GP}^{49}$

This would allow a more accurate selection of patients for $\mathrm{Hp}$, as well as better preoperative planning. Additionally, molecular mechanisms associated with the different tumor-liver interfaces may lead to the development of targeted therapies. $44,46,50$
Nevertheless, our study has some limitations: the study population is small, from a single institution and the design is retrospective, which may reflect some variation in patient selection. Moreover, the long period inevitably leads to distinct technical approaches to resection of both primary tumor and LM, different imaging methods for diagnosis, staging and surveillance and heterogeneity in exposure to different kinds of chemotherapy regimens different criteria in the use of neoadjuvant or adjuvant chemotherapies. We recommend adjuvant chemotherapy as a contributor to the cure of these patients. In addition, there may have been some discrepancy in the access to positron emission tomography, magnetic resonance imaging and laparoscopy throughout the period of the study. Finally, as the treatment of GC was performed in many institutions the principles of treatment and surveillance applied may differ, possibly affecting the timing of the $\mathrm{Hp}$ and in the survival times.

The authors acknowledge that the study population is small for solid conclusions to be withdrawn. However, most series of patients undergoing hepatectomy for GCLM are small, with size of study population under 18 patients in many cases. ${ }^{16,51-53}$

The authors recognize that the long study period, during which major advances in medical oncology, radiology and even surgical technique were witnessed, may induce some bias in the interpretation of results. An example of this, are the evolving indications for hepatectomy and perioperative chemotherapy. Nonetheless, in spite of these facts and of the small study sample, the innovative aspect of this work is the finding of a definite impact of the desmoplastic GP of gastric cancer liver metastases in the patient survival. This has not previously been documented and confirms this important histological marker as a distinct phenotypic feature of the tumor-host interaction.

In conclusion, we can safely say that $\mathrm{Hp}$ in selected patients with GCLM can prolong survival, as illustrated in our cohort by two patients surviving more than 10 years after Hp. Selection of patients with less aggressive GC, indolent dissemination and, a favorable tumor-host response is likely to be the key to grant survival advantage. ${ }^{10}$ By looking at both GC and metastases in detail, we suggest that the characteristics of tumor microenvironment - tumor-related, host-related and tumor-host reciprocal interface - are crucial to prognosis. In fact, metachronous presentation and response to neoadjuvant chemotherapy might reflect a more indolent tumoral behavior. 
Desmoplastic GP is, for the first time, reported as an independent prognostic factor after Hp for GCLM. Further research into the biological mechanism driving this phenotype is mandatory.

\section{Abbreviations}

Hp, hepatectomy; GCLM, gastric carcinoma liver metastases; GP, growth pattern; GC, gastric carcinoma; TTNI, tumor thickness at the tumor-normal interface; TRG, tumor regression grade; CRLM, colorectal cancer liver metastases; AFC, Association Française de Chirurgie; PHLF, posthepatectomy liver failure; WHO, World Health Organization; NOS, non-otherwise specified; OS, overall survival; DFS, disease-free survival.

\section{Statement of Ethics}

Study was approved by the institutional ethics committee ("Comissão de Ética do Centro Hospitalar e Universitário de Coimbra" - number CHUC-127-19).

\section{Author Contributions}

MJT performed study concepts, data acquisition, analysis and interpretation statistical analysis and manuscript preparation. RCO performed study concept and design, data acquisition, analysis and interpretation and manuscript preparation. RM, MS, CC, ES and LF performed acquisition of data. PT was responsible for acquisition of data and analysis. BC and MAC were responsible for manuscript editing, data acquisition, analysis and interpretation. JGT performed study conception, manuscript editing and critical review. HA performed study concept and design, data acquisition, analysis and interpretation, manuscript preparation and editing. All authors have drafted or written, or substantially revised or critically reviewed the article. All authors have agreed to submit the manuscript to this journal, reviewed and agreed on all versions of the article before submission, during revision, the final version accepted for publication, and any significant changes introduced at the proofing stage and agree to take responsibility and be accountable for the contents of the article.

\section{Funding}

No funding was necessary.

\section{Disclosure}

There is no conflict of interest. This article is partially based on Maria José Temido's Master Thesis in Medicine ("Clinical and pathological factors of prognosis after hepatectomy for gastric cancer liver metastases"). The manuscript was rewritten and refined in order to be substantially different and henceforth acceptable for publication. The Thesis is cited whenever it is appropriate.

\section{References}

1. Ferlay J, Soerjomataram I, Dikshit R, et al. Cancer incidence and mortality worldwide: sources, methods and major patterns in GLOBOCAN 2012. Int J Cancer. 2014;136:359-386. doi:10.1002/ ijc. 29210

2. Metastatic Cancer. National cancer institute. 2017. Available from: https:/www.cancer.gov/types/metastatic-cancer. Accessed November $2,2020$.

3. D’Angelica M, Gonen M, Brennan MF, Turnbull AD, Bains M, Karpeh M. Patterns of initial recurrence in completely resected gastric adenocarcinoma. Ann Surg. 2004;240(5):808-816. doi:10.1097/ 01.sla.0000143245.28656.15

4. Kodera Y, Sano T. Japanese gastric cancer treatment guidelines 2014 (ver. 4). Gastric Cancer. 2017;20(1):1-19. doi:10.1007/s10120-0160622-4

5. Zhang W, Yu Y, Fang YFY, Wang YCK, Cui Y. Systemic chemotherapy as a main strategy for liver metastases from gastric cancer. Clin Transl Oncol. 2015;17(11):888-894. doi:10.1007/ s12094-015-1321-z

6. Liao Y, Peng N, Long D, Yu P, Zhang S, Zhong J. Hepatectomy for liver metastases from gastric cancer: a systematic review. BMC Surg. 2017;17(1):1-7. doi:10.1186/s12893-017-0215-0

7. Kinoshita T, Kinoshita T, Saiura A, Esaki M, Sakamoto H, Yamanaka T. Multicentre analysis of long-term outcome after surgical resection for gastric cancer liver metastases. Br J Surg. 2015;102 (1):102-107. doi:10.1002/bjs.9684

8. Tatsubayashi T, Tanizawa Y, Miki Y, et al. Treatment outcomes of hepatectomy for liver metastases of gastric cancer diagnosed using contrast-enhanced magnetic resonance imaging. Gastric Cancer. 2017;20(2):387-393. doi:10.1007/s10120-016-0611-7

9. Xi H, Liang W, Li J, et al. Evaluation of hepatectomy and palliative local treatments for gastric cancer patients with liver metastases: a propensity score matching analysis. Oncotarget. 2017;8 (37):61861-61875. doi:10.18632/oncotarget.18709

10. Temido MJ, Oliveira RC, Alexandrino H Clinical and pathological factors of prognosis after hepatectomy for gastric cancer liver metastases - is desmoplastic growth the key to longer survival? 2018. Available from: https://estudogeral.sib.uc.pt/bitstream/10316/82542/ 1/MariaTemidoFinal.pdf. Accessed November 2, 2020.

11. Hwang S-E, Yang D-H, Kim C-Y. Prognostic factors for survival in patients with hepatic recurrence after curative resection of gastric cancer. World J Surg. 2009;33(7):1468-1472. doi:10.1007/s00268009-0034-2

12. Kerkar SP, Kemp CD, Avital I. Liver resections in metastatic gastric cancer. HPB. 2010;12(9):589-596. doi:10.1111/j.1477-2574.2010. 00224.x

13. Garancini M, Uggeri F, Degrate L, et al. Surgical treatment of liver metastases of gastric cancer: is local treatment in a systemic disease worthwhile? HPB. 2012;14(3):209-215. doi:10.1111/j.14772574.2011.00428.x

14. Wang Y, Shen K, Ling J, et al. Prognostic analysis of combined curative resection of the stomach and liver lesions in 30 gastric cancer patients with synchronous liver metastases. BMC Surg. 2012;12:20. doi:10.1186/1471-2482-12-20

15. Ohkura Y, Shinohara H, Haruta S, et al. Hepatectomy offers superior survival compared with non-surgical treatment for $\leq 3$ metastatic tumors with diameters $<3 \mathrm{~cm}$ from gastric cancer: a Retrospective Study. World J Surg. 2015;39(11):2757-2763. doi:10.1007/s00268-015-3151-0 
16. Markar SR, Mikhail S, Malietzis G, et al. Influence of surgical resection of hepatic metastases from gastric adenocarcinoma on long-term survival: systematic review and pooled analysis. Ann Surg. 2016;263(6):1092-1101. doi:10.1097/SLA.0000000000001542

17. Song A, Zhang X, Yu F, Li D, Shao W, Zhou Y. Surgical resection for hepatic metastasis from gastric cancer: a multi-institution study. Oncotarget. 2017;54:71147-71153. doi:10.18632/oncotarget.16705

18. Montagnani F, Crivelli F, Aprile G, et al. Long-term survival after liver metastasectomy in gastric cancer: systematic review and meta-analysis of prognostic factors. Cancer Treat Rev. 2018;69:11-20. doi:10.1016/j.ctrv.2018.05.010

19. Maru DM, Kopetz S, Boonsirikamchai P, et al. Tumor thickness at the tumor-normal interface: a novel pathologic indicator of chemotherapy response in hepatic colorectal metastases. Am J Surg Pathol. 2010;34(9):1287-1294. doi:10.1097/PAS.0b013e3181eb2f7b

20. Rubbia-Brandt L, Giostra E, Brezault C, et al. Importance of histological tumor response assessment in predicting the outcome in patients with colorectal liver metastases treated with neo-adjuvant chemotherapy followed by liver surgery. Ann Oncol. 2006;18 (2):299-304. doi:10.1093/annonc/mdl386

21. Jørgensen JT, Hersom M. HER2 as a prognostic marker in gastric cancer - a systematic analysis of data from the literature. $J$ Cancer. 2012;3(1):137-144. doi:10.7150/jca.4090

22. Vermeulen PB, Colpaert C, Salgado R, et al. Liver metastases from colorectal adenocarcinomas grow in three patterns with different angiogenesis and desmoplasia. $J$ Pathol. 2001;195:336-342. doi:10.1002/path.966

23. Falcão D, Alexandrino $H$, Caetano Oliveira R, et al. Histopathologic patterns as markers of prognosis in patients undergoing hepatectomy for colorectal cancer liver metastases - pushing growth as an independent risk factor for decreased survival. Eur J Surg Oncol. 2018;44 (8):1212-1219. doi:10.1016/j.ejso.2018.03.023

24. Fernández Moro C, Bozóky B, Gerling M. Growth patterns of colorectal cancer liver metastases and their impact on prognosis: a systematic review. BMJ Open Gastroenterol. 2018;5(1):e000217. doi:10.1136/bmjgast-2018-000217

25. van Dam P-J, van der Stok EP, Teuwen L-A, et al. International consensus guidelines for scoring the histopathological growth patterns of liver metastasis. Br J Cancer. 2017;117(10):1427-1441. doi:10.1038/bjc.2017.334

26. Oliveira RC, Alexandrino H, Cipriano MA, Tralhão JG. Liver metastases and histological growth patterns: biological behavior and potential clinical implications - another path to individualized medicine? J Oncol. 2019;2019:1-6. doi:10.1155/2019/6280347

27. Adam R, Chiche L, Aloia T, et al. Hepatic resection for noncolorectal nonendocrine liver metastases. Ann Surg. 2006;244(4):524-535. doi:10.1097/01.sla.0000239036.46827.5f

28. Martins J, Alexandrino H, Oliveira R, et al. Sinusoidal dilation increases the risk of complications in hepatectomy for CRCLM protective effect of bevacizumab and diabetes mellitus, serum gamma-glutamyltranspeptidase as predictive factor. Eur J Surg Oncol. 2016;42(5):713-721. doi:10.1016/j.ejso.2016.02.017

29. Dindo D, Demartines N, Clavien P. Classification of surgical complications. Ann Surg. 2004;240(2):205-213. doi:10.1097/01. sla.0000133083.54934.ae

30. Balzan S, Belghiti J, Farges O, et al. The " 50-50 criteria " on postoperative day 5: an accurate predictor of liver failure and death after hepatectomy. Ann Surg. 2005;242:824-829. doi:10.1097/01. sla.0000189131.90876.9e

31. Rahbari NN, Garden OJ, Padbury R, et al. Posthepatectomy liver failure: a definition and grading by the International Study Group of Liver Surgery (ISGLS). Surgery. 2011;149:713-724. doi:10.1016/j. surg.2010.10.001
32. Koch M, Garden OJ, Padbury R, et al. Bile leakage after hepatobiliary and pancreatic surgery: a definition and grading of severity by the International Study Group of Liver Surgery. Surgery. 2011;149:680-688. doi:10.1016/j.surg.2010.12.002

33. Rahbari NN, Garden OJ, Padbury R, et al. Post-hepatectomy haemorrhage: a definition and grading by the International Study Group of Liver Surgery (ISGLS). HBP. 2011;13:528-535. doi:10.1111/ j.1477-2574.2011.00319.x

34. Bosman F, Carneiro F, Hruban RH, Theise N. WHO Classification of Tumours of the Digestive System. IARC Press; 2010.

35. Japanese Gastric Cancer Association. Japanese classification of gastric carcinoma: 3rd english edition. Gastric Cancer. 2011;14 (2):101-112. doi:10.1007/s10120-011-0041-5

36. Sano K, Yamamoto M, Mimura T, et al. Outcomes of 1639 hepatectomies for non-colorectal non-neuroendocrine liver metastases: a multicenter analysis. J Hepatobiliary Pancreat Sci. 2018;25 (11):465-475. doi:10.1002/jhbp.587

37. Ministrini S, Solaini L, Cipollari C, et al. Surgical treatment of hepatic metastases from gastric cancer. Updates Surg. 2018;59 (2):177-184. doi:10.1023/a:1006398401352

38. Ito $\mathrm{H}$, Are $\mathrm{C}$, Gonen $\mathrm{M}$, et al. Effect of postoperative morbidity on long-term survival after hepatic resection for metastatic colorectal cancer. Ann Surg. 2008;247(6):994-1002. doi:10.1097/ SLA.0b013e31816c405f

39. Aloia TA, Zimmitti G, Conrad C, Gottumukalla V, Kopetz S, Vauthey J-N. Return to Intended Oncologic Treatment (RIOT): a novel metric for evaluating the quality of oncosurgical therapy for malignancy. J Surg Oncol. 2012;17(10):1310-1314. doi:10.1038/ nm.2451.A

40. Vibert E, Pittau G, Gelli M, et al. Actual incidence and long-term consequences of posthepatectomy liver failure after hepatectomy for colorectal liver metastases. Surg (United States). 2014;155 (1):94-105. doi:10.1016/j.surg.2013.05.039

41. Barnhill R, Vermeulen P, Daelemans S, et al. Replacement and desmoplastic histopathological growth patterns: a pilot study of prediction of outcome in patients with uveal melanoma liver metastases. $J$ Pathol Clin Res. 2018;4(4):227-240. doi:10.1002/cjp2.105

42. Van Den Eynden GG, Majeed AW, Illemann M, et al. The multifaceted role of the microenvironment in liver metastasis: biology and clinical implications. Cancer Res. 2013;73(7):2031-2043. doi:10.1158/0008-5472.CAN-12-3931

43. Venkatesan S, Swanton C, Taylor BS, Costello JF. Treatment-induced mutagenesis and selective pressures sculpt cancer evolution. Cold Spring Harb Perspect Med. 2017;7(8):a026617. doi:10.1101/cshperspect.a026617

44. Fu Y, Du P, Zhao J, Hu C, Qin Y, Huang G. Gastric cancer stem cells: mechanisms and therapeutic approaches. Yonsei Med J. 2018;59 (10):1150-1158. doi:10.3349/ymj.2018.59.10.1150

45. Joerger AC, Fersht AR. The p53 pathway: origins, inactivation in cancer, and emerging therapeutic approaches. Annu Rev Biochem. 2016;85(1):375-404. doi:10.1146/annurev-biochem-060815014710

46. Lazaris A, Amri A, Petrillo SK, et al. Vascularization of colorectal carcinoma liver metastasis: insight into stratification of patients for anti-angiogenic therapies. J Pathol Clin Res. 2018;4(3):184-192. doi: $10.1002 /$ cjp 2.100

47. Frentzas S, Simoneau E, Bridgeman VL, et al. Vessel co-option mediates resistance to anti-angiogenic therapy in liver metastases. Nat Med. 2017;22(11):1294-1302. doi:10.1038/nm.4197

48. Eefsen RL, Engelholm L, Alpizar-Alpizar W, et al. Inflammation and uPAR-expression in colorectal liver metastases in relation to growth pattern and neo-adjuvant therapy. Cancer Microenviron. 2015;8 (2):93-100. doi:10.1007/s12307-015-0172-z 
49. Oliveira RC, Alexandrino H, Cipriano MA, Alves FC, Tralhão JG. Predicting liver metastases growth patterns: current status and future possibilities. Semin Cancer Biol. 2020. doi:10.1016/j. semcancer.2020.07.007

50. Levine AJ. Targeting therapies for the $\mathrm{p} 53$ protein in cancer treatments. Annu Rev Cancer Biol. 2019;3(1):21-34. doi:10.1146/ annurev-cancerbio-030518-055455

51. Nonaka Y, Hiramatsu K, Kato T, et al. Evaluation of hepatic resection in liver metastasis of gastric cancer. Indian J Surg Oncol. 2019;10 (1):204-209. doi:10.1007/s13193-018-0827-6
52. Choi SB, Song J, Kang CM, et al. Surgical outcome of metachronous hepatic metastases secondary to gastric cancer. Hepatogastroenterology. 2010;57(97):29-34.

53. Fukami Y, Kaneoka Y, Maeda A, et al. Adjuvant hepatic artery infusion chemotherapy after hemihepatectomy for gastric cancer liver metastases. Int $J$ Surg. 2017;46:79-84. doi:10.1016/j. ijsu.2017.08.578

\section{Publish your work in this journal}

Cancer Management and Research is an international, peer-reviewed open access journal focusing on cancer research and the optimal use of preventative and integrated treatment interventions to achieve improved outcomes, enhanced survival and quality of life for the cancer patient.
The manuscript management system is completely online and includes a very quick and fair peer-review system, which is all easy to use. Visit http://www.dovepress.com/testimonials.php to read real quotes from published authors. 